\title{
Visualizing Computer-Based Activity on Ambient Displays to Reduce Sedentary Behavior at Work
}

\author{
Hans Brombacher \\ Industrial Design Department, Eindhoven University of \\ Technology, the Netherlands. \\ Steven Vos \\ Industrial Design Department, Eindhoven University of \\ Technology, the Netherlands.School of Sport Studies, \\ Fontys University of Applied Sciences, the Netherlands.
}

\begin{abstract}
Workplace health interventions have predominantly been designed around visualizations of physical activity data in the work routine. Yet, contextual factors, such as computer-based activity, appears to be crucial to support healthier behaviors at work. In this research, we explore the effect of visualizing computer-based activity to prompt physical activity at work, through desktop-based ambient displays. Based on our prototypes Yamin and Apphia, we conducted an exploratory qualitative user study in a lab setting with office workers $(\mathrm{N}=16)$. Results showed that visualizing one's computer-based activity could potentially increase the awareness, self-reflection, and social interactions for individuals to become physically active. With our findings, we discuss design implications for using computer activity data in a physical form as a motivational factor to encourage physically active workstyles. We present directions for future field studies to gain further insights on this topic.
\end{abstract}

\section{CCS CONCEPTS}

- Human-centered computing $\rightarrow$ Human computer interaction (HCI); HCI design and evaluation methods.

\section{KEYWORDS}

Physical activity, Office environment, Computer-based activity data, Ambient displays, Data visualization

\section{ACM Reference Format:}

Hans Brombacher, Xipei Ren, Steven Vos, and Carine Lallemand. 2020. Visualizing Computer-Based Activity on Ambient Displays to Reduce Sedentary Behavior at Work. In 32ND AUSTRALIAN CONFERENCE ON HUMANCOMPUTER INTERACTION (OzCHI '20), December 02-04, 2020, Sydney, NSW, Australia. ACM, New York, NY, USA, 5 pages. https://doi.org/10.1145/ 3441000.3441022

Permission to make digital or hard copies of part or all of this work for personal or classroom use is granted without fee provided that copies are not made or distributed for profit or commercial advantage and that copies bear this notice and the full citation on the first page. Copyrights for third-party components of this work must be honored For all other uses, contact the owner/author(s).

OzCHI '20, December 02-04, 2020, Sydney, NSW, Australia

(c) 2020 Copyright held by the owner/author(s).

ACM ISBN 978-1-4503-8975-4/20/12.

https://doi.org/10.1145/3441000.3441022

\author{
Xipei Ren \\ Industrial Design Department, Eindhoven University of \\ Technology, the Netherlands. \\ Carine Lallemand \\ Industrial Design Department, Eindhoven University of \\ Technology, the Netherlands. HCI Research Group, \\ University of Luxembourg, Esch-sur-Alzette, Luxembourg \\ c.e.lallemand@tue.nl
}

\section{INTRODUCTION AND RELATED WORK}

In the workplace, information and communication technologies play a significant role in reducing physical movement. This inactive and sedentary-focused behavior is associated with diseases such as type II diabetes, cardiovascular diseases, and breast cancer [5, 17]. Digital services have provided individuals with convenient access to information and efficient team interactions without breaking the workflow in majorly "seated" office jobs. As measures to reduce unhealthy inactive workstyles, several interventions have been using office workers' physical activity data [11], such as step count [7], sitting time [29], heart rate [31] and postures [32] to raise awareness or prompt breaks. However, it is challenging to promote physical activity in the workplace without triangulating physical activity data and contextual information [27]. For instance, the majority of office work routines are dominated by computer-based tasks, which can significantly influence workers' behavioral intention to move more [4, 9]. Visualizing these working behaviors may support self-awareness and self-reflection on opportunities and intentions for being active during the workday [13]. Current interventions using computer activity data as an input are often displaying outputs under the form of dashboards or prompts on the computer screen $[1,13,28,36]$. Physical and tangible designs addressing office sedentary behavior exist, ranging from office furniture, lamps, or wearables $[10-12,20]$. Yet as shown in the scoping review by Damen et al. [11], none of the artefacts reviewed used computer data as an input measure (preferring step counts, heartrate and motion).

In this project, we investigate whether and how visualization of computer data can act as a motivational factor to trigger physically active behaviors at the office. We present a preliminary researchthrough-design study exploring the role of real-time computerbased activity data in relation to physical activity through ambient displays. Ambient displays provide "physical ambient cues, signals, and alerts that present socially meaningful events in the data as changes in the physical environment" $[18,19]$. Through their physical properties, they offer the advantage of increasing awareness and participation [18]. Prior research also hints at the potential of using ambient technologies for a persuasive purpose [33], for instance at the workplace by nudging employees to take a break (Breakaway by Jafarinaimi et al. [22]) or to use the stairs rather than the elevator (Light installations by Rogers et al. [33]). Other 

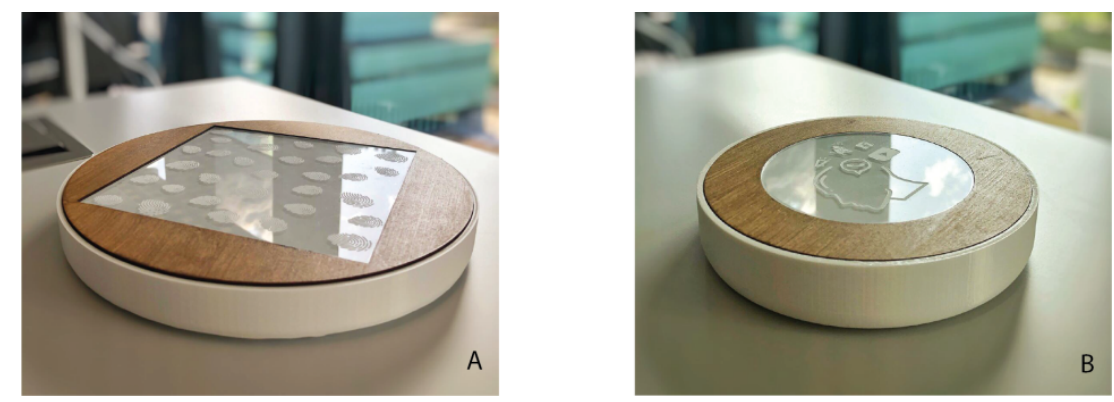

Figure 1: Yamin (a) and Apphia (b), two ambient desktop devices that visualize computer-activity

notable design examples using ambient displays to nudge office workers into moving more are Movelamp [15], which indicates the employee's recent activity level, Lightsit [31] which visualizes unhealthy sitting behavior or posture, or the Healthbar [29] which helps users break their sitting behavior. These examples use and embody several forms of physical activity data, yet none relate physical activity to computer activity data.

We designed two ambient desktop devices that visualize data based on trackpad activity (Figure 1a Yamin) and the duration of "productive" computer time (Figure 1b Apphia), data sources often used in the field of affective computing [35]. As shown in Figure 2, we mapped computer activity data to LED matrixes in our devices. We used these artefacts as research probes during a lab evaluation study with 16 participants, to evaluate their applicability to the context and discuss design opportunities. We contribute to the field of Human-Computer interaction (HCI) by exploring the role of visualizing computer data in relation to physical activity data through ambient displays embedding innovative characteristics (in their visualization, metrics, and intended impact). The produced knowledge is relevant for both the development of design interventions and research setups on the timely topic of physical activity in the office environment.

\section{PROTOTYPING YAMIN AND APPHIA}

We started our design process by brainstorming the type of data that could be visualized in order to raise awareness and, eventually, prompt people to take more breaks and be more active at work. We designed two artefacts of similar aspect yet representing distinct perspectives. First, Yamin was developed to visualize computer activity as measured via trackpad data. A heatmap-like lighting display is presented on Yamin (Figure 2a) to synthesize the historical data of cursor positions on a laptop. A running character is represented through green LEDs. It becomes less and less prominent as the cursor data lights up and takes over the visualization. We used the Processing environment to acquire computer use data. Second, Apphia uses time tracking software data from RescueTime ${ }^{\circledR}$ as a visualization of daily productivity. It tracks how people spend time on their computer and categorizes it to show which apps or website are most distracting. As a popular platform offering effective metrics and an API, RescueTime was appropriate for our study. Apphia leverages a circle of light spots with different color codes (Figure $2 b$ ) to represent focused time (green) and distracted time (red). To do so, RescueTime sends the number of non-productive minutes to the Particle cloud. A Particle Photon subscribed to this data string compares the data with a set threshold (e.g. 20 min of non-productive work). The data collected through our artefacts could (and already is in the case of RescueTime) be displayed on the computer itself, yet following research on ambient interfaces $[18,19]$ we hypothesized that a physical display placed visibly on one's desk would have a different impact. The designs were developed as ambient interfaces to increase awareness and participation through their physical properties [18, 21, 23]. Making information visible in a social setting like the office environment [22] is another characteristic of both artefacts. As visualizations of computer-related data, mouse activity and productivity (as measured through RescueTime) were selected for their ability to trigger reflection and change in the working routine. On Yamin (Figure 2), the running character depicted, which slowly fades in the background, raises awareness on the user's sedentary behavior. On Apphia, the visualization is not directly related to sedentary behavior. Our prototypes act as research probes to investigate what meaning workers make of the visualization of their computer-based working behaviors and if it can raise awareness to decrease sedentary behaviors in the office routine.

\section{USER STUDY}

We conducted a user study involving sixteen office workers ( 9 males, 7 females, average age 37.9) in a lab setting (Figure 3). Participants signed a consent form and did not receive any incentive. The user study entailed a showcase and testing session and a follow-up semi-structured interview. First, we presented a live demo of basic interaction mechanisms using the prototypes and a video showing the visualization in-situ. Participants had the opportunity to interact with the prototypes for 15 minutes. The follow-up interview aimed at understanding participants' first experience and attitude towards the design concepts. Questions prompted them to a) reflect on the visualized link between their computer data and their physical activity data, b) compare the two forms of data presented and the meaning they attribute to them and finally c) envision how these ambient displays would impact their physical (in)activity at work. Interviews were recorded, transcribed and analyzed using thematic analysis [6]. 

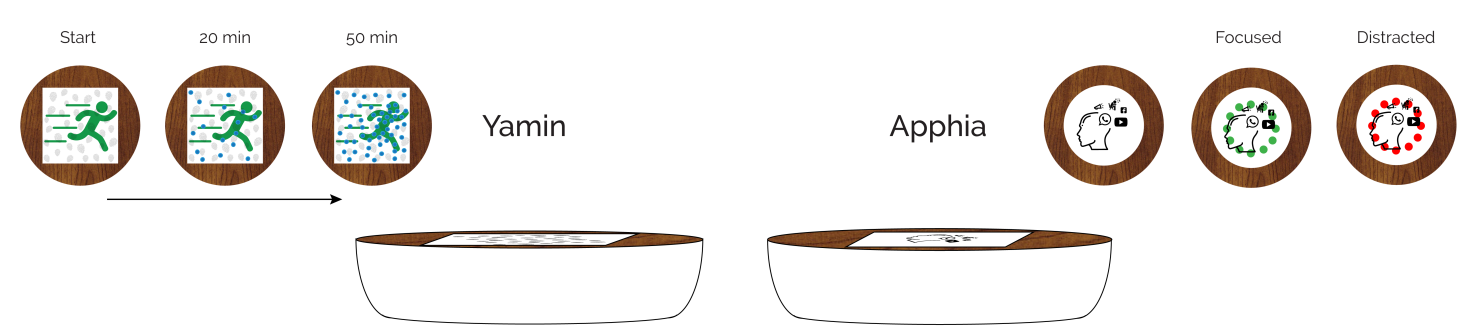

Figure 2: Computer data visualization of Apphia and Yamin. Apphia visualizes with an $8 x 8$ matrix if an individual is focused or distracted, based on their computer use. Yamin visualizes with a $16 \times 16$ matrix if an active person being progressively covered by mouse activity (blue dots) over time.

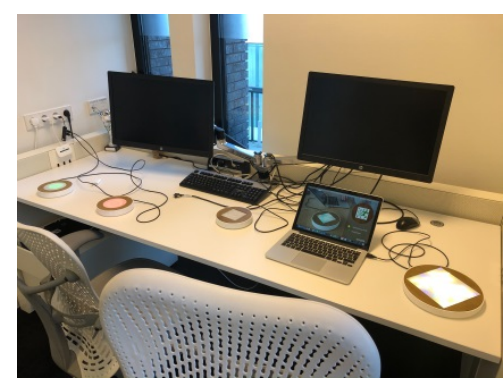

Figure 3: Research setup of the lab study

\section{RESULTS}

Three themes emerged from the interview analysis, hinting at how ambient data visualizations of computer-based activity may contribute to office vitality.

\subsection{Self-Awareness of Unhealthy Working Behaviors}

Participants (11 out of 16) indicated a behavioral intention to reduce sedentary behavior due to the awareness of their computer-based activity. This was mostly seen with Apphia, visualizing productive working time: "I think that many people sit behind their computers unconsciously for a long time without knowing that their productivity is decreasing." (P2) "I like it because the device now indicates that you are no longer focused and that you maybe should take a break." (P15) and "It can also be a confrontation. If you get red feedback that you are wondering, what have I actually done in the last hour. I could have been more active." (P1). In contrast, Yamin was perceived as less influential to remind being active at work. Two participants expressed that visualizing the trackpad activity reveals only limited or ambiguous information and thus could not support users in self-awareness. "It may be that when you are reading a lot, your mouse use is very low." (P13). It was however appreciated that the data was expressed as a process: "I like the mouse activity because it shows a process over a longer period." (P6). Showing the process also gave participants a feeling of deserving a break because they worked hard for a time period: "I can see for myself how I'm doing. . . I deserve to be distracted when it is completely full [green character covered by mouse data] or around $80 \%$ that I have worked hard and can have a break." (P2).

\subsection{Awareness through Ambient Displays}

Instead of a screen-based activity dashboard, Yamin and Apphia visualize computer activity on desk-based ambient devices to convey concise information to the user (mentioned by 8 participants). The "physical" property of the artefacts was perceived as more effective, increasing a sort of ambient awareness during work, than traditional interventions using visualizations on the computer itself. As $\mathrm{P} 3$ indicated, "It is confronting because with the computer you click it away quickly, but you cannot ignore this. But it is positive because it indicates it and you can go for a walk." Being physical also served the purpose of moving people away from screens "It doesn't all have to be digital. I've been looking at my computer all day and this is a kind of external trigger that helps you remember." (P14), "You can't click it away [comparing Yamin to a digital intervention] so I think that this will help. I think this is positive." (P9), "I like it, because otherwise everything stays on the screen and this gives it a slightly different atmosphere" (P10) and "It's on your desk so you can't just ignore it or put it away, I like that. Because normally on an app you swipe it away." (P11).

\subsection{Social Considerations}

Due to the collective nature of office work, the data visualized on Yamin and Apphia was linked by participants to social aspects in the workplace. For instance, the setup was seen as an opportunity to trigger social support for office vitality "I would find it useful to find out if someone is available for a conversation or needs to be active. Or maybe they have been sitting at the computer for too long and you can help." (P4) On the flip side, two participants also stressed the privacy issues that could arise: "The disadvantage is that it is not private. It can therefore be less pleasant because sometimes you have busier or more relaxed periods during work, and this is now visible." (P6). "If my neighbor goes red all the time then that might be a bit privacy sensitive..." (P13).

\section{DISCUSSION AND FUTURE WORK}

This study was set out to explore the effect of ambient displays visualizing computer activity as a mean to reduce physical inactivity in the work routine. The main goal was to support self-awareness and self-reflection on opportunities and behavioral intentions for being active during the workday. Through prototyping and conducting a first study of Yamin and Apphia, we learned that it could support self-awareness about one's working pattern, and perhaps prompt 
an active work break. The visualization of productive working time was considered more useful than the visualization of trackpad activity, which was deemed less comprehensive and ambiguous about the tasks achieved. In Yamin and Apphia the visualization of computer activity was represented in a physical form rather than a digital intervention on the computer. The designs contributed to an experience of ambient awareness, which can easily fit into the busy work routine [20] while needing only little cognitive resources as suggested by the concept of Ambient Persuasive Technology by Ham and Midden [16]. This might support office workers in a seamless transition from the increasingly sedentary nature of computer-supported work to spontaneous active breaks.

Our user study also brought up several discussions about the social implications of physical visualizations of computer activity in the office context. On the one hand, the proposed designs have potential to increase social interactions to encourage physical activity and healthy behaviors among co-workers. On the other hand, the social aspects of our designs should be thought of carefully to avoid negative social stimulus (e.g., unfriendly competition) and potential privacy issues regarding personal data sharing. The office environment consists of individuals with different levels of competitiveness [14]. The balance should therefore be found where the computer activity is presented for a heterogeneous group of office workers while giving individuals a clear motivation. From a privacy perspective, the design team has additionally been questioning and reflecting on the potential misuse of employees' data by their organization. While Apphia visualized participants' preferred type of data, the clear and publicly accessible visualization of productive versus non-productive time triggered ethical considerations. In this regard, translating data that was traditionally displayed privately on one's screen into a desktop visible device should be done with the highest caution. More user research is needed to get insights into scenarios where displaying this data would not be harmful to users [2]. It might for instance include environments that are even more sedentary due to the absence of social contact, such as freelance work and remote home office. Additionally, the use of red and green colors in the current Apphia prototype might convey the wrong message about focused and distracted time, where the former would be signaled as more appropriate than the other, potentially increasing productivity pressure over a healthy balance of tasks and activities.

Being exploratory in nature, the present study presents several limitations which should be addressed in future work. First, the relatively short duration of the study in a lab setting, focused on first impressions and attitudes towards the design concepts, limits the ecological validity of our findings [25]. It does not inform the user experience of our design proposals in an actual office work setting over a prolonged time. Second, contrary to previously published interventions [11], our devices do not measure direct data about sedentary behavior or physical (in)activity of individuals. Future work could consider triangulating data about computer activity with data about sedentary behavior, using off-the-shelf sensing products or wearables, such as fitness trackers [7] or office furniture $[3,8,31]$. Third, our artefacts are physical ambient displays yet do not include interactivity in the interaction. Previous work by Hornecker and Buur [18] has emphasized tangible and reconfigurable aspects as key to empower users and encourage discoverability and sensemaking of the data presented. Other authors [33] highlighted playfulness and the ability to lure and intrigue, as well as aesthetics [22] as effective characteristics of ambient displays for behavior change. We thus aim at iterating on our designs, in order to trigger more meaningful explorations and sensemaking.

In a future research study, we will deploy our artefacts in the field as experiential probes [30] to investigate the short and mid-term effects of our designs in-situ. Scaling up a field intervention on a sufficient sample of office workers will require additional prototypes, a difficulty often encountered in interventions using physical artefacts instead of digital tools. Two successive rounds of 6-weeks studies in office environments are thus foreseen with a total of 8 prototypes ( $\mathrm{N}=16$ office workers). While novelty and discoverability will be key elements of the user experience in the first days or weeks of use [24], this timeframe will allow to study usefulness and effects on behavior beyond the familiarization stage. During deployment (starting 2 weeks before the intervention as a baseline) we will provide participants with activity trackers (Fitbit @ $\odot$ wristbands) in order to measure the effect of our artefacts on sedentary behavior and to compare it to activity logs of the devices. The evaluation of user experience of ambient systems is however challenging as users do not have to proactively interact with the system [34]. We will use semi-structured interviews and a diary study [26] to understand workers' experiences over time. Of particular interest will be how users individually place the ambient design in their work environment and how this can impact its effectiveness. The social interactions triggered by the devices will also be analyzed. In line with a previous study [7], we uncovered here an interesting perspective on how ambient visualization of work behavior can trigger social processes. We aim at further investigating how social interactions between colleagues can support office workers' adoption of healthier and more active workstyles. By exploring the role of visualizing computer data in relation to physical activity data through ambient displays, we hope to inspire design interventions and research setups to address sedentary behavior at work.

\section{ACKNOWLEDGMENTS}

We would like to thank our colleague Daphne Menheere for her input during the co-creation sessions.

\section{REFERENCES}

[1] Lars Louis Andersen, Emil Sundstrup, Marianne Boysen, Markus Due Jakobsen, Ole Steen Mortensen, and Roger Persson. 2013. Cardiovascular Health Effects of Internet-Based Encouragements to Do Daily Workplace Stair-Walks: Randomized Controlled Trial. Journal of Medical Internet Research 15, 6 (2013). DOI:http: //dx.doi.org/10.2196/jmir.2340

[2] Julio Angulo, Simone Fischer-Hübner, Tobias Pulls, and Erik Wästlund. 2015. Usable Transparency with the Data Track. Proceedings of the 33rd Annual ACM Conference Extended Abstracts on Human Factors in Computing Systems - CHI EA 15 (2015). DOI:http://dx.doi.org/10.1145/2702613.2732701

[3] Dechristian França Barbieri et al. 2017. Sit-Stand Tables With Semi-Automated Position Changes: A New Interactive Approach for Reducing Sitting in Office Work. IISE Transactions on Occupational Ergonomics and Human Factors 5, 1 (2017), 39-46. DOI:http://dx.doi.org/10.1080/24725838.2016.1259191

[4] Toufique Bharmal, Marc Hassenzahl, and Matthias Laschke. 2020. From Intentions to Successful Action: Supporting the Creation and Realization of Implementation Intentions. In Extended Abstracts of the $2020 \mathrm{CHI}$ Conference on Human Factors in Computing Systems (CHI EA '20). Association for Computing Machinery, New York, NY, USA, 1-8. DOI:https://doi.org/10.1145/3334480.3383018

[5] Stuart J.H. Biddle. 2007. Sedentary Behavior. American Journal of Preventive Medicine 33, 6: 502-504. https://doi.org/10.1016/j.amepre.2007.08.002 
[6] Virginia Braun and Victoria Clarke. 2006. Using thematic analysis in psychology. Qualitative Research in Psychology3, 2 (2006), 77-101. DOI:http://dx.doi.org/10. 1191/1478088706qp063oa

[7] Hans Brombacher, Dennis Arts, Carl Megens, and Steven Vos. 2019. Stimulight: exploring social interaction to reduce physical inactivity among office workers Extended Abstracts of the 2019 CHI Conference on Human Factors in Computing Systems - CHI EA 19 (2019). DOI:http://dx.doi.org/10.1145/3290607.3313094

[8] Xu, L., Chen, G., Wang, J., Shen, R., \& Zhao, S. (2012). A sensing cushion using simple pressure distribution sensors. 2012 IEEE International Conference on Multisensor Fusion and Integration for Intelligent Systems (MFI) doi:10.1109/mfi.2012.6343048

[9] Chia-Fang Chung, Nanna Gorm, Irina A. Shklovski, and Sean Munson. 2017. Finding the Right Fit. Proceedings of the $2017 \mathrm{CHI}$ Conference on Human Factors in Computing Systems - CHI 17 (2017).

[10] Ida Damen, Anika Kok, Vink Bas, Hans Brombacher, Steven Vos, and Carine Lallemand. The Hub : Facilitating Walking Meetings through a Network of Interactive Devices. In Companion Publication of the 2020 ACM Designing Interactive Systems Conference. https://doi.org/10.1145/3393914.3395876

[11] Ida Damen, Hans Brombacher, Carine Lallemand, Rens Brankaert, Aarnout Brom bacher, Pieter Van Wesemael and Steven Vos. 2020. A Scoping Review of Digital Tools to Reduce Sedentary Behavior or Increase Physical Activity in Knowledge Workers. International Journal of Environmental Research and Public Health 2020; 17(2):499. DOI: https://doi.org/10.3390/ijerph17020499

[12] Ida Damen, Lidewij Heerkens, Annabel van den Broek, Kimberly Drabbels, Olga Cherepennikova, Hans Brombacher, and Carine Lallemand. 2020. PositionPeak: Stimulating Position Changes During Meetings. In Extended Abstracts of the 2020 CHI Conference on Human Factors in Computing Systems https://doi.org/ $10.1145 / 3334480.3383054$

[13] Maria José Ferreira, Ana Karina Caraban, and Evangelos Karapanos. 2014. Breakout. Proceedings of the extended abstracts of the 32nd annual ACM conference on Human factors in computing systems - CHI EA 14 (2014). DOI:http: //dx.doi.org/10.1145/2559206.

[14] Thomas D. Fletcher, Debra A. Major, and Donald D. Davis. 2008. The interactive relationship of competitive climate and trait competitiveness with workplace attitudes, stress, and performance. Journal of Organizational Behavior, 29, 7 (2008), 899-922. DOI:http://dx.doi.org/10.1002/job.503

[15] Jutta Fortmann, Tim Stratmann, Susanne Boll, Benjamin Poppinga, and Wilko Heuten. 2013. Make Me Move at Work! An Ambient Light Display to Increase Physical Activity. Proceedings of the ICTs for improving Patients Rehabilitation Research Techniques (2013).

[16] Jaap Ham and Cees Midden. 2010. Ambient Persuasive Technology Needs Little Cognitive Effort: The Differential Effects of Cognitive Load on Lighting Feedback versus Factual Feedback. In Persuasive Technology. Springer Berlin Heidelberg, 132-142. https://doi.org/10.1007/978-3-642-13226-1_14

[17] Jameson K. Hirsch, Danielle Molnar, Edward C. Chang, and Fuschia M. Sirois. 2014 Future orientation and health quality of life in primary care: vitality as a mediator Quality of Life Research 24, 7: 1653-1659. https://doi.org/10.1007/s11136-0140901-7

[18] Eva Hornecker, and Jacob Buur. "Getting a grip on tangible interaction: a framework on physical space and social interaction." In Proceedings of the SIGCHI conference on Human Factors in computing systems, pp. 437-446. ACM, 2006.

[19] Steven Houben, Connie Golsteijn, Sarah Gallacher, Rose Johnson, Saskia Bakker Nicolai Marquardt, Licia Capra, and Yvonne Rogers. 2016. Physikit. In Proceedings of the 2016 CHI Conference on Human Factors in Computing Systems. https: //doi.org/10.1145/2858036.2858059

[20] Yitong Huang, Steve Benford, and Holly Blake. 2019. Digital Interventions to Reduce Sedentary Behaviors of Office Workers: Scoping Review. Journal of Medical
Internet Research21, 2 (2019). DOI:http://dx.doi.org/10.2196/11079

[21] Hiroshi Ishii, and Brygg Ullmer. "Tangible bits: towards seamless interfaces between people, bits and atoms." In Proceedings of the ACM SIGCHI Conference on Human factors in computing systems, pp. 234-241. ACM, 1997.

[22] Nassim Jafarinaimi, Jodi Forlizzi, Amy Hurst, and John Zimmerman. 2005. Breakaway. CHI 05 extended abstracts on Human factors in computing systems - CHI 05(2005). DOI:http://dx.doi.org/10.1145/1056808.1057063

[23] Yvonne Jansen, Pierre Dragicevic, Petra Isenberg, Jason Alexander, Abhijit Karnik, Johan Kildal, Sriram Subramanian, and Kasper Hornbæk. "Opportunities and challenges for data physicalization." In CHI 2015-Proceedings of the SIGCHI Conference on Human Factors in Computing Systems. 2015.

[24] Evangelos Karapanos, John Zimmerman, Jodi Forlizzi, and Jean-Bernard Martens. 2009. User experience over time. In Proceedings of the 27th international conference on Human factors in computing systems - CHI 09. https://doi.org/10.1145/ 1518701.1518814

[25] Carine Lallemand, Vincent Koenig, V. 2017. Lab Testing Beyond Usability: Challenges and Recommendations for Assessing User Experiences. Journal of Usability Studies, 12 (3).

[26] Carine Lallemand. 2012. Dear diary: Using diaries to study User Experience, User Experience Magazine, 11, 3. Retrieved from http://uxpamagazine.org/dear-diaryusing-diaries-to-study-user-experience

[27] Ian Li, Anind K. Dey, and Jodi Forlizzi. 2012. Using context to reveal factors that affect physical activity. ACM Transactions on Computer-Human Interaction 19, 1 (January 2012), 1-21. DOI:http://dx.doi.org/10.1145/2147783.2147790

[28] Yuhan Luo, Bongshin Lee, Donghee Yvette Wohn, Amanda L. Rebar, David E. Conroy, and Eun Kyoung Choe. 2018. Time for Break. Proceedings of the 2018 CHI Conference on Human Factors in Computing Systems - CHI 18 (2018). DOI:http://dx.doi.org/10.1145/3173574.3173701

[29] Victor Mateevitsi, Khairi Reda, Jason Leigh, and Andrew Johnson. 2014. The health bar. Proceedings of the 5th Augmented Human International Conference on - AH 14 (2014). DOI:http://dx.doi.org/10.1145/2582051.2582072

[30] Michel Peters and Carl Megens. 2014. Experiential design landscapes: how to design for behavior change, towards an active lifestyle (2014). DOI:https://doi. org/10.6100/IR771700

[31] Xipei Ren, Bin Yu, Yuan Lu, Biyong Zhang, Jun Hu, and Aarnout Brombacher. 2019. LightSit: An Unobtrusive Health-Promoting System for Relaxation and Fitness Microbreaks at Work. Sensors19, 9 (2019), 2162. DOI:http://dx.doi.org/10. 3390/s19092162

[32] Xipei Ren, Bin Yu, Yuan Lu, Yu Chen, and Pearl Pu. 2018. HealthSit: Designing Posture-Based Interaction to Promote Exercise during Fitness Breaks. International Journal of Human-Computer Interaction 35, 10 (July 2018), 870-885. DOI:http://dx.doi.org/10.1080/10447318.2018.1506641

[33] Yvonne Rogers, William R. Hazlewood, Paul Marshall, Nick Dalton, and Susanna Hertrich. 2010. Ambient influence. In Proceedings of the 12th ACM international conference on Ubiquitous computing. https://doi.org/10.1145/1864349.1864372

[34] Ewald Strasser, Astrid Weiss, Thomas Grill, Sebastian Osswald, and Manfred Tscheligi. 2012. Combining Implicit and Explicit Methods for the Evaluation of an Ambient Persuasive Factory Display. In Lecture Notes in Computer Science. Springer Berlin Heidelberg, 113-128. https://doi.org/10.1007/978-3-642-34898$3 \_8$

[35] Jianhua Tao and Tieniu Tan. 2005. Affective Computing and Intelligent Interaction. Lecture Notes in Computer Science (2005). DOI:http://dx.doi.org/10.1007/ 11573548

[36] Yunlong Wang and Harald Reiterer. 2019. The Point-of-Choice Prompt or the Always-On Progress Bar? Extended Abstracts of the 2019 CHI Conference on Human Factors in Computing Systems - CHI EA 19 (2019).DOI:http://dx.doi.org/ $10.1145 / 3290607.3313050$ 